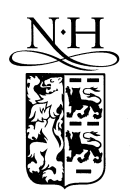

ELSEVIER

\title{
Pulse dynamics in absorber dominated optical cavities
}

\author{
G. Shaulov ${ }^{\mathrm{a}, 1}$, I. Gabitov ${ }^{\mathrm{b}}$, J.W. Haus ${ }^{\mathrm{a}, 2}$ \\ ${ }^{a}$ Department of Physics, Rensselaer Polytechnic Institute, Troy, NY 12180-3590, USA \\ b Theoretical Division T-7, MS B284, Los Alamos National Laboratory, Los Alamos, NM 87545, USA
}

Received 25 September 1998; received in revised form 1 December 1998; accepted 17 March 1999

\begin{abstract}
We investigate optical field dynamics in lasers cavities dominated by a saturable absorber. We found that for a specific parameter range the solitary wave-type solutions exist and can be expressed in analytic form, including a new gray-pulse solution. Stability of these solitary waves is discussed and numerically examined. (C) 1999 Published by Elsevier Science B.V. All rights reserved.
\end{abstract}

The design of passive mode-locked lasers incorporates an element that has the action of a saturable absorber. Recently ultrashort pulses generated in fiber lasers using a multiple quantum well (MQW) saturable absorber to generate pulses [1-3]. In these lasers the absorber properties dominate the cavity dynamics. This distinguishes them from other mode-locked lasers designed with strong soliton shaping [4].

The theory of modelocking is based on the master equation [4,5] derived under the condition that nonlinear changes to the intra-cavity pulse must be small per round-trip and a fast saturable absorber action. The carrier dynamics is adiabatically eliminated due to the sub-picosecond material response time and pulse widths of order ten picoseconds. To obtain analytic results we use the form of a complex Ginzburg-Landau equation, where the saturable absorber nonlinear contributions are approximated by an expansion of the field amplitude to fifth order; this has limited quantitative application, but the results are qualitatively the same. The equation has a form:

$$
\frac{\partial A}{\partial z}=\left(D_{g}+i D\right) \frac{\partial^{2} A}{\partial t^{2}}-\gamma_{1} A+\left(\gamma_{3}+i \delta\right)|A|^{2} A-\gamma_{5}|A|^{4} A,
$$

where $A$ is the optical field complex amplitude, $z$ is the spatial coordinate scaled to the cavity length, $t$ is the local time, $\gamma_{1}=l-g>0$ is the net linear loss in the cavity ( $g, l$ represent gain and loss per round trip), $D$ is the cavity dispersion, $D_{g}$ is the gain (or filter) bandwidth limitation in the cavity, $\delta$ is the cavity self-phase

\footnotetext{
${ }^{1}$ Present address: Tyco Submarine Systems, 250 Industrial Way, Eatontown, NJ 07724, USA

${ }^{2}$ Corresponding author. E-mail: hausjj@rpi.edu
} 
modulation and $\gamma_{3}, \gamma_{5}$ represent nonlinear gain and loss due to the absorber, which may be defined through the linear absorption coefficient $q_{0}$ and absorber saturation power $P_{s}$ :

$$
D=-\frac{\beta_{2} L_{c}}{2}, \quad D_{g}=\frac{g}{\Omega_{g}^{2}}+\frac{1}{\Omega_{f}^{2}}, \quad \gamma_{3}=\frac{q_{0}}{P_{s}}, \quad \gamma_{5}=\frac{q_{0}}{P_{s}^{2}},
$$

with $L_{c}$ - cavity length, $\beta_{2}$ - group velocity dispersion and $\Omega_{g(f)}-$ gain (filter) bandwidth.

For operation of a fiber laser designed with multiple quantum wells [1,2], the absorption dominates the cavity dynamics and the operating parameters are well outside the soliton regime. We restrict our analysis to this situation. The following scaling is useful

$$
\psi=\sqrt{1 / P_{s}} A, \quad Z=q_{0} z, \quad T=\sqrt{q_{0} / D_{g}} t ;
$$

with parameters

$$
\alpha=\gamma_{1} / q_{0}, \quad \mu=D / D_{g}, \quad \nu=\delta / \gamma_{3} .
$$

Eq. (1) then has the form

$$
\psi_{Z}=(1+i \mu) \psi_{T T}-\alpha \psi+(1+i \nu)|\psi|^{2} \psi-|\psi|^{4} \psi
$$

The subscripts on the complex field amplitude $\psi$ denote derivatives with respect to scaled spatial and temporal variables. Eq. (3) belongs to a class of quintic complex Ginzburg-Landau equations and were intensively studied due to its wide application in different fields [6-9].

The parameters of the fiber laser designed with 25-75 quantum wells in the saturable absorber element as reported in Ref. [1,2] are the following: $L_{c}=10 \mathrm{~m}, P_{s}=12 \mathrm{~W}, q_{0}=0.2, \Omega_{f}=0.785 \mathrm{THz}$, which corresponds to the $1 \mathrm{~nm}$ Bragg grating bandwidth (the gain bandwidth is $30 \mathrm{~nm}$ and can be neglected). Then the physical coefficients are $D=0.05 \mathrm{ps}^{2}, D_{g}=1.62 \mathrm{ps}^{2}, \delta=2.6 \times 10^{-5} \mathrm{~mW}^{-1}$ and $\gamma_{3}=1.67 \times 10^{-2} \mathrm{~mW}^{-1}$, i.e. $\mu=3.08 \times 10^{-2}$ and $\nu=1.56 \times 10^{-3}$. Since $\mu, \nu \ll 1$, in first approximation we ignore the imaginary terms. Eq. (3) with real coefficients can be represented in variational form

$$
\frac{\partial \psi}{\partial Z}=-\frac{\delta F}{\delta \psi^{*}},
$$

where $F=\int \mathscr{F} d T$ is analogous to a free energy with its density

$$
\mathscr{F}=\mathscr{T}-U=\left|\psi_{T}\right|^{2}+\alpha|\psi|^{2}-\frac{1}{2}|\psi|^{4}+\frac{1}{3}|\psi|^{6} .
$$

$\mathscr{T}$ denotes the kinetic energy density contribution, i.e. the first term on the right hand side, and $U$ represents the potential energy density. Minima (maxima) of free energy $F$ correspond to stationary stable (unstable) states.

It is known $[9,10]$ that for systems of this type travelling wave solutions are determined by the transitions either from one stable state to the other or from the unstable state to the stable one. The direction of the travelling waves is defined by an energy minimization principle. We will consider only transitions from one stable state with higher energy to other one with smaller energy.

The shape of potential $U$ is parameterized by $\alpha$. As shown in Fig. 1, it possesses two maxima (corresponding to stationary stable states) that have equal heights for the special case $\alpha=3 / 16$. The zero amplitude stable state has higher (lower) energy than the second stable state at greater amplitude for $\alpha<3 / 16(\alpha>3 / 16)$.

Traveling wave solutions of Eq. (3) are obtained by assuming $\psi(Z, T)=\psi(T-c Z)=\psi(\tau)$, where $c$ has a meaning of difference between inverse travelling wave velocity and inverse group velocity of the light, and the one to be determined. Eq. (3) becomes an ordinary differential equation with the coefficient $c$ appearing as a 

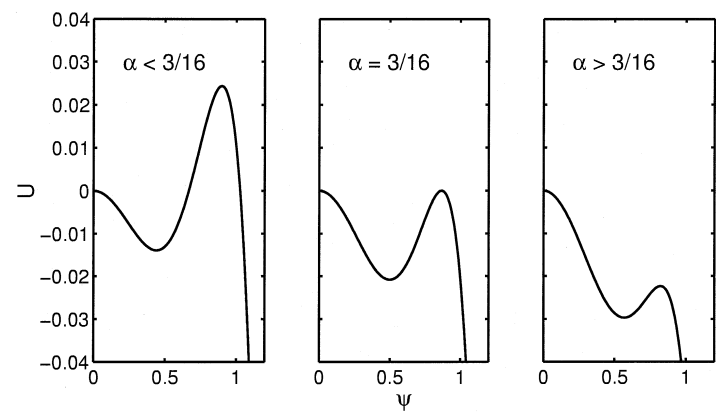

Fig. 1. Plot of the potential for the conservative equations of motion and three values of the parameter $\alpha$.

linear damping coefficient in an otherwise conservative system. Let $\psi(\tau)=v(\tau) \exp (i \phi(\tau))$ where $v$ and $\phi$ are real functions, and define $u=v_{\tau} / v$ and $\Omega=\phi_{\tau}$, then Eq. (3) is rewritten as the system of differential equations

$$
v_{\tau}=v u, \quad u_{\tau}=-u^{2}-c u+\Omega^{2}+\alpha-v^{2}+v^{4}, \quad \Omega_{\tau}=-\Omega(2 u+c) .
$$

Extrema of the free energy correspond to the stationary points (SP) ( $\left.v_{\tau}=u_{\tau}=\Omega_{\tau}=0\right)$ of Eq. (3) as shown on Fig. 2. SP can exist only at $\Omega=0$, therefore phase of the travelling wave $\phi=\phi_{0}=$ const.

In the plane $\Omega=0$ two SP have coordinates $\left(v_{0}^{ \pm}, 0\right)$, and the other two have coordinates $\left(0, u_{0}^{ \pm}\right)$, where the amplitude values are

$$
v_{0}^{ \pm}=\sqrt{\frac{1 \pm \sqrt{1-4 \alpha}}{2}}, \quad u_{0}^{ \pm}=-\frac{c}{2} \pm \sqrt{\left(\frac{c}{2}\right)^{2}+\alpha} .
$$

Three SP are always saddle points (correspond to stable states - free energy minima) and the fourth stationary point, $\left(v_{0}^{-}, 0\right)$, is either a stable (unstable) node $(c>0(c<0))$ or an elliptic point $(c=0)$ (unstable state - free energy maximum).

Time dependent solutions generate trajectories in the phase plane that connect stationary points. For instance, transient solutions occur when the laser is turned on or off; these are represented by trajectories III $\rightarrow$ I or I $\rightarrow$ IV given by dashed lines in Fig. 2. The transient solutions have the shape of a front or kink; they can be

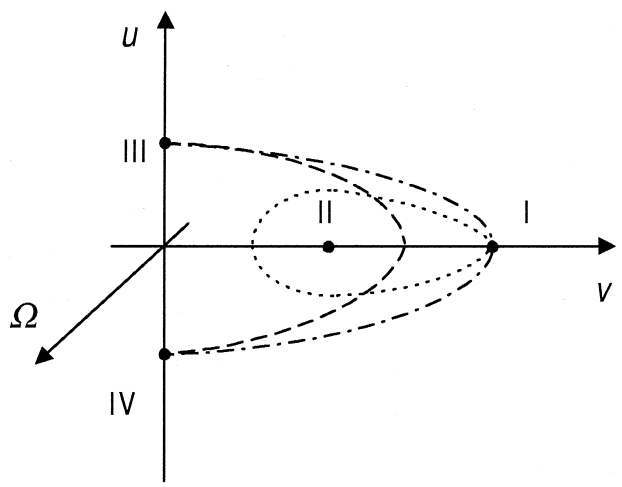

Fig. 2. Invariant plane stationary points. Stability near the stationary points is discussed in the text. 

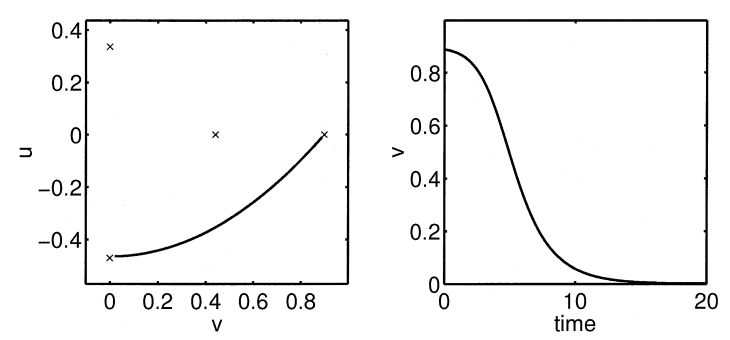

Fig. 3. The transient solution from point I to point IV in Fig. 2 for $\alpha=5 / 32$. The left side outlines the trajectory and the right side is the corresponding field amplitude.

analytically described. For example, the shape of the front corresponding to the transition $\left(0, u_{0}^{+}\right) \rightarrow\left(v_{0}^{+}, 0\right)$ has the form

$$
\psi(\tau)=\frac{v_{0}^{+} \mathrm{e}^{i \phi_{0}}}{\left(1+\mathrm{e}^{-2 a \tau}\right)^{1 / 2}}, \quad(\tau=T-c Z),
$$

where $\psi \rightarrow v_{0}^{+} \mathrm{e}^{i \phi_{0}}$ at $\tau \rightarrow \infty$ and $\psi \rightarrow 0$ at $\tau \rightarrow-\infty$ (see Ref. [9] and references there). Here $a=-\left(v_{0}^{+}\right)^{2} / \sqrt{3}$, $c=(2 \sqrt{1-4 \alpha}-1) / \sqrt{3}$ (where $0<\alpha<1 / 4)$ and $\phi_{0}$ - constant phase. This solution is demonstrated in Fig. 3. The parameter $c$ vanishes for $\alpha=3 / 16$, i.e. front (kink) velocity coincides with the group velocity. In addition to the transient solutions, we found that the system also has pulse and gray pulse-like solutions at $c=0$.

Modelocked lasers provide coherent pulses of light that ideally have a zero background. Point III to point IV transition, denoted by a dashed line in Fig. 2, is a solitary pulse solution. It is only formed when $c=0$ and $\alpha<3 / 16$. An example is shown in Fig. 4; the trajectory in the plane $\Omega=0$ is depicted on the left and the right side shows the corresponding field amplitude. The analytic form of the solution can be derived using method described in Ref. [11]

$$
\psi(T)=\left[\frac{4 \alpha}{1+\sqrt{1-16 / 3 \alpha} \cosh (\sqrt{4 \alpha} T)}\right]^{1 / 2} \mathrm{e}^{i \phi_{0}}
$$

Gray pulse solutions corresponds to the transition starting and ending at stable state $v_{0}^{+} ; c=0$ and $\alpha>3 / 16$. A sample trajectory is drawn with a dash-dotted line in Fig. 2; it begins and ends on point I. Fig. 5 shows a
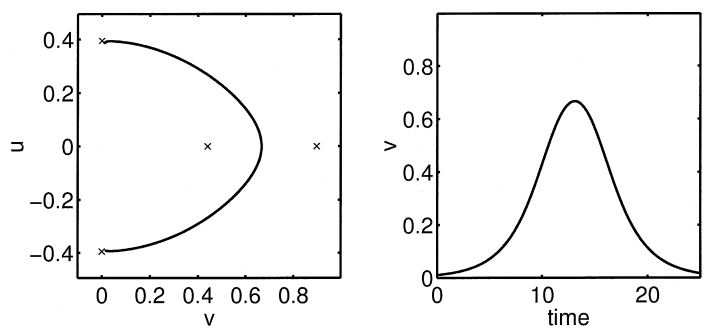

Fig. 4. The pulse solution for $\alpha=5 / 32$. The left side is the trajectory in the $\Omega=0$ plane and the right side is the the corresponding field amplitude. 

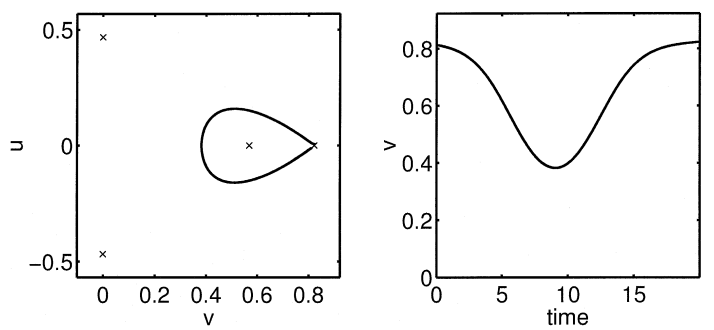

Fig. 5. Gray pulse solution for $\alpha=7 / 32$. The left side is the trajectory in the $\Omega=0$ plane and the right side is the corresponding field amplitude.

numerical example; again with the transition orbit shown on the left and the field amplitude on the right. The analytic solution is

$$
\psi(T)=\left[1-\frac{3\left(2\left(v_{0}^{+}\right)^{2}-1\right)}{2\left(v_{0}^{+}\right)^{2}-\left(3 / 2-2\left(v_{0}^{+}\right)^{2}\right)\left(1-\cosh \left[\sqrt{2\left(v_{0}^{+}\right)^{2}\left(2\left(v_{0}^{+}\right)^{2}-1\right)} T\right]\right)}\right]^{1 / 2} v_{0}^{+} \mathrm{e}^{i \phi_{0}} .
$$

The gray pulse solution is a solitary pulse on a CW background. This solution has not been presented in earlier studies.

Linear stability analysis reveals that transient solutions are stable, whereas the solitary pulse and gray pulse solutions in general are unstable. Clearly, stable pulses are the most interesting objects, but the unstable solutions can grow extremely slowly. In fact, the unstable solutions close to $\alpha=3 / 16$ undergo slow changes. Near this value transient and pulse solutions can coexist and their trajectories are close to one another, so that there is only a small distinction between them.

Existence of stable transient solutions leads to the possibility of pulse-like objects being generated. For $\alpha<3 / 16$ leading and trailing edges of a pulse propagate in opposite directions. An expanding plateau forms on top of this "pulse". This plateau corresponds to the lowest energy state. However, as $\alpha \rightarrow 3 / 16$ from below, and the leading and tailing edges are close enough to each other, so that their velocities vanish; then the pulse approaches the solution given by Eq. (8). Fig. 6 demonstrates an initial sech-pulse solution rapidly evolving to a pulse, $\alpha=3 / 16$, which is composed of two slightly displaced transient on-off solutions. At this point the distinction between pulse and transient solutions is lost. In the case when $\alpha \rightarrow 3 / 16$ from above we have observed similar behavior: well-like expanding transient on-off solutions approaching a gray pulse.

The pulse shape given by Eq. (8) depends on the parameter $\alpha$. At the values $\alpha=3 / 16$ and $\alpha=0$, the pulse transforms to a constant field or the pulse becomes infinitely broad. For convenience we introduced a new parameter $\epsilon$ in the following way $\alpha=3 / 16-\epsilon$. Fig. 7 shows the pulse width dependence on $\epsilon$ corresponding to intermediate values of the parameter $\alpha$. The minimum value of the pulse width is approximately $15 \mathrm{ps}$. ( $\alpha \sim 0.15$ and $\epsilon \sim 0.0375$ ). This result is qualitatively the same as found in experiments on modelocked fiber lasers in Ref. [2], where output pulses of duration 7-50 ps were observed. Numerical simulations yield what appears to be a stable pulse when $\alpha=3 / 16 \pm \epsilon$ with $\epsilon=10^{-3}-10^{-6}$, at least over a propagation distance of up to $z=1000$. In this case, the parameter $c$ differs from zero by a correction of order $\epsilon$. This parameter can be experimentally adjusted by changing the cavity losses at mirrors and the output coupler or by adjusting the gain through the pump laser. Analysis shows that perturbations of (gray) pulse solutions due to dispersion and self-phase modulation in the cavity (i.e. small values of $\mu$ and $\nu$ in Eq. (3)) do not change their stability properties. Future research will be devoted to examining pulse stability. Our simulations show that perturbed solutions approach a solitary wave, which establishes a region for stabilized pulse solutions. Despite the lack of soliton shaping mechanisms in our modelocked laser model, there are parameter regimes where quasi-stable pulses can be formed. 


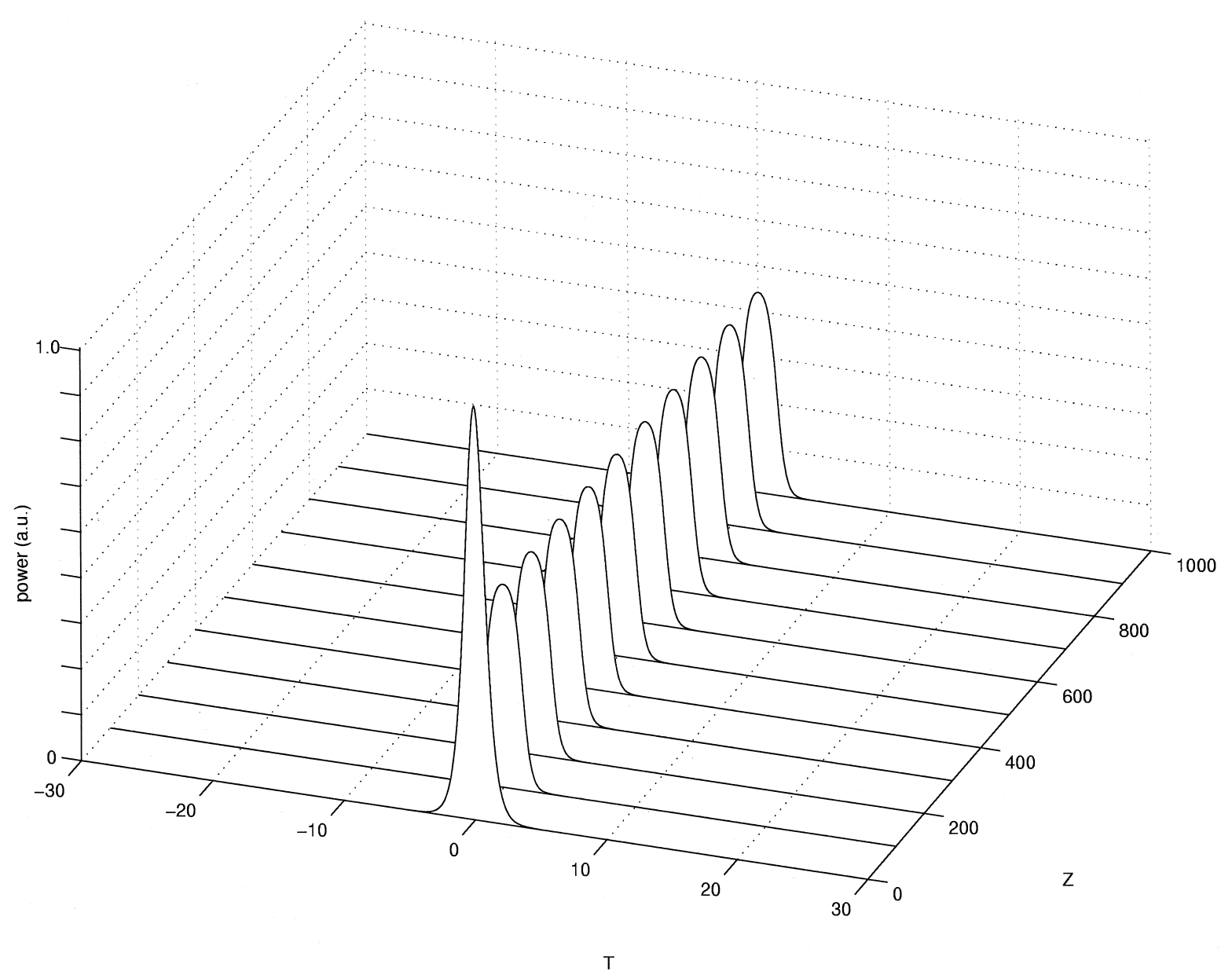

Fig. 6. An initial sech-pulse evolves to a solitary-wave solution. $\alpha=3 / 16$; the front velocities are zero for this case.

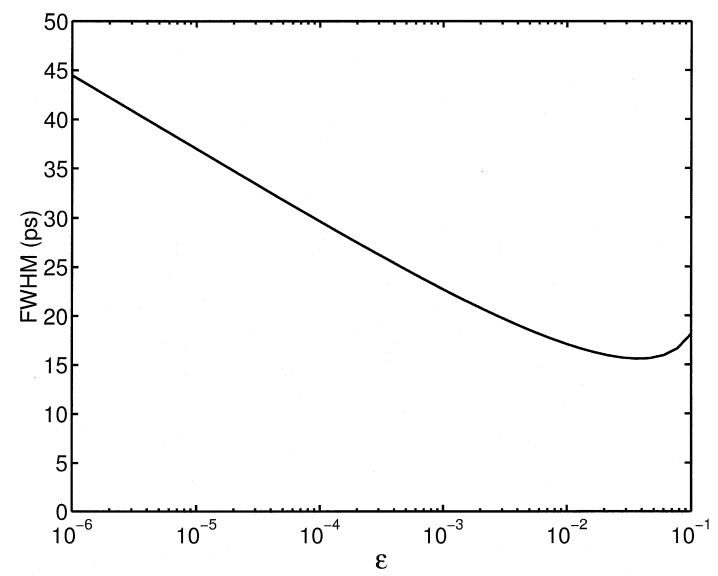

Fig. 7. Pulse width versus parameter $\epsilon$. 


\section{Acknowledgements}

This work was supported by a National Science Foundation Grant DMS-9510728 and by Rome Laboratories Grant F30602-96-2-0056. The authors also acknowledge many helpful discussions with Profs. G. Kovacic, V. Roytburd, and M. Levi.

\section{References}

[1] M. Hayduk, Ph.D. Thesis, Cornell University, 1997.

[2] W. Kaechele, Ph.D. Thesis, Rensselaer Polytechnic Institute, 1997.

[3] B.C. Barnett, L. Rahman, M.N. Islam, Y.C. Chen, P. Brattacharya, W. Riha, K.V. Reddy, A.T. Howe, K.A. Stair, H. Iwasmura, S.R. Friberg, T. Mukai, Optics Lett. 20 (1995) 471.

[4] H.A. Haus, in: I.N. Duling, III (Ed.), Compact Sources of Ultrashort Pulses, Cambridge Univ. Press, Cambridge, 1995.

[5] F.X. Kartner, I.D. Jung, U. Keller, IEEE J. Quant. Electron. 2 (1996) 540.

[6] N.N. Akhmediev, A. Ankiewicz, Solitons: Nonlinear Pulses and Beams, Chapman and Hall, London, 1997; J.M. Soto-Crespo, N.N. Akhmediev, V.V. Afanasjev, J. Opt. Soc. Am. B 13 (1996) 1439.

[7] A.A. Nepomnyashchy, In: A.V. Gaponov-Grekhov et al. (Eds.), Nonlinear waves I, Dynamics and evolution, 103-110, Springer-Verlag, Berlin et al., 1989; B.A. Malomed, A.A. Nepomnyashchy, Phys. Rev. A 42 (1990) 6009.

[8] J.A. Powell, A.C. Newell, C.K.R.T. Jones, Phys. Rev. A 44 (1991) 3636; C.K.R.T. Jones, T.M. Kapitula, J.A. Powell, Proc. R. Soc. Edinburg A 116 (1990) 193.

[9] W.van Saarloos, P.C. Hohenberg, Physica D 56 (1992) 303.

[10] V.G. Kamensky, S.V. Manakov, JETP Lett. 45 (1987) 638.

[11] M. Florjanczyk, L. Gagnon, Phys. Rev. A 41 (1990) 4478. 